\title{
Circadian regulation of low density lipoprotein receptor promoter activity by CLOCK/BMAL1, Hes1 and Hes6
}

\author{
Yeon-Ju Lee ${ }^{1}$, Dong-Hee Han ${ }^{1}$, \\ Youngmi Kim Pak ${ }^{1,2}$ and Sehyung Cho ${ }^{1,2,3}$ \\ ${ }^{1}$ Department of Neuroscience and Neurodegeneration Control \\ Research Center \\ Kyung Hee University \\ Seoul 130-701, Korea \\ ${ }^{2}$ Department of Physiology \\ Kyung Hee University School of Medicine \\ Seoul 130-701, Korea \\ ${ }^{3}$ Corresponding author: Tel, 82-2-961-0984; \\ Fax, 82-2-969-6343; E-mail, sehyung@khu.ac.kr \\ http://dx.doi.org/10.3858/emm.2012.44.11.073
}

Accepted 22 August 2012

Available Online 30 August 2012

Abbreviations: BMAL1, brain and muscle arnt-like 1; CLOCK, circadian locomotor output cycle kaput; CRY1, cryptochrome 1; Hes1, hairy and enhancer of split 1; Hes6, hairy and enhancer of split 6; IDL, intermediate density lipoprotein; LDLR, low density lipoprotein receptor; PER1, period homolog 1; RHT, retinohypothalamic tract; SCN, suprachiasmatic nucleus; SRE, sterol regulatory element

\begin{abstract}
Low density lipoprotein receptor (LDLR) plays an important role in the cholesterol homeostasis. We examined the possible circadian regulation of LDLR and mechanism(s) underlying it. In mice, blood glucose and plasma triglyceride, total and high density lipoprotein cholesterol varied distinctively throughout a day. In addition, LDLR mRNA oscillated in the liver in a functional clock-dependent manner. Accordingly, analysis of human LDLR promoter sequence revealed three putative $\mathrm{E}$-boxes, raising the possible regulation of LDLR expression by E-box-binding transcription factors. To test this possibility, human LDLR promoter reporter constructs were transfected into HepG2 cells and the effects of CLOCK/BMAL1, Hes1, and Hes6 expression were analyzed. It was found that positive circadian transcription factor complex CLOCK/BMAL1 upregulated human LDLR promoter activity in a serumindependent manner, while Hes family members Hes1
\end{abstract}

and Hes6 downregulated it only under serum-depleted conditions. Both effects were mapped to proximal promoter region of human LDLR, where mutation or deletion of well-known sterol regulatory element (SRE) abolished only the repressive effect of Hes1. Interestingly, hes6 and hes1 mRNA oscillated in an anti-phasic manner in the wild-type but not in the per $^{-1 /}$ per2 ${ }^{-/}$mouse. Comparative analysis of mouse, rat and human hes6genes revealed that three E-boxes are conserved among three species. Transfection and site-directed mutagenesis studies with hes6 reporter constructs confirmed that the third E-box in the exon IV is functionally induced by CLOCK/BMAL1. Taken together, these results suggest that LDLR expression is under circadian control involving CLOCK/BMAL1 and Hes family members Hes1 and Hes6.

Keywords: ARNTL transcription factors; circadian rhythm; CLOCK proteins; HES1 protein, human; HES6 protein, human; receptors, LDL

\section{Introduction}

The lives of most organisms living on earth are governed by the repetitive changes of day and night generated by rotation of the earth on its axis. The circadian (circa: around, dies: day) clock enables an organism to anticipate environmental changes throughout a day, to ensure proper timing for biological processes, and to improve the efficiency of biological energy uses. Circadian rhythms govern many aspects of physiological and metabolic functions including the sleep-wake cycle, feeding behavior, body temperature, as well as cardiovascular, endocrine, gastrointestinal, hepatic and renal functions.

In mammals, circadian rhythms are endogenously generated by 'central/master pacemaker' in the suprachiasmatic nucleus (SCN) of the anterior hypothalamus, which orchestrates many 'peripheral/slave oscillators' present in numerous peripheral tissues. The central clock is entrained by external time cues called 'Zeitgeber', such as light. In photic entrainment, SCN receives inputs through retinohypothalamic tract (RHT) and synchronizes the peripheral clocks in peripheral tissues by the neural and humoral 
outputs (Moore et al., 1983; Panda and Hogenesch, 2004; Noh et al., 2011). In addition to the light, another important Zeitgeber for the peripheral tissues is food (Stokkan et al., 2001; Ishida et al., 2005; Yoon et al., 2012).

The molecular machinery of circadian clock is composed of complex positive and negative transcriptional and translational feedback loops. The positive clock components, CLOCK and BMAL1 (MOP3) form heterodimer and activate transcription of target genes by binding to the E-box sequence (CANNTG) on their regulatory sequence. The negative components, PERs and CRYs containing E-box on their promoters are induced by CLOCK/ BMAL1, accumulate in the cytoplasm, translocate to the nucleus with timed delay, and inhibit the action of CLOCK/BMAL1. This feedback loop generates transcriptional and translational fluctuations and allows various rhythms of enzymes activity, hormone secretion and many physiological aspects with $\sim 24$ $\mathrm{h}$ period even under constant conditions (Dunlap, 1999; Roenneberg et al., 2003).

Dysregulation of circadian rhythms in human may result in pathological conditions. For example, shift workers are subject to unusual daily cycle so that their disruption of biological rhythms leads to disorders in physiology and metabolism. Indeed, many studies have reported increased obesity, type Il diabetes, and cardiovascular diseases in shift workers (Knutsson, 2003; Ha et al., 2005).

The relation between circadian dysregulation and pathophysiology can be mechanistically studied in mice. By mutation or knockout of core clock components, the role of each clock component in numerous physiological processes can be understood. In these mutated mice, various pathological conditions including metabolic syndrome, abnormal gluconeogenesis, apoptosis/cancer development, abnormal lipogenesis and retinal degeneration/blind has been reported (reviewed in Ko and Takahashi, 2006). For example, Clock/Clock mutant mice become obese and develop metabolic syndrome with hepatic steatosis, hyperglycaemia, hypertriglyceridaemia, hypercholesterolaemia and hyperleptinaemia (Turek et al., 2005). This reveals that circadian regulation is linked to metabolism and dysregulation of circadian rhythms can contribute to metabolic diseases.

The cholesterol level in blood is related to several metabolic diseases such as hypercholesterolaemia, high blood pressure, atherosclerosis and cardiovascular diseases. According to recent studies (Whitmer et al., 2008), it also triggers dementia and decline of renal function. Thus, proper regulation of blood cholesterol is important for health and wellbeing.

Cholesterol is an important component for the manufacturing of bile acids, steroid hormones, cell membrane and several fat-soluble vitamins, and it is either taken via dietary sources or synthesized de novo within the body. Since cholesterol is insoluble in blood, it is transported in the circulatory system by lipoprotein carriers, such as chylomicrons, very low density lipoproteins (VLDLs), intermediate density lipoproteins (IDLs), low density lipoprotein (LDLs), and high density lipoproteins (HDLs). Among these, LDL is the major carrier of cholesterol in the blood and high levels of LDL-cholesterol in the blood is related to several diseases. LDL molecules can be oxidized and taken up by macrophages, which become engorged and form foam cells. These cells often become trapped in the walls of blood vessels and contribute to atherosclerotic plaque formation. These plaques are one of the main causes of heart attacks, strokes, and other serious medical problems. Thus, proper regulation of LDL-cholesterol within the blood is important for the prevention of atherosclerosis. In various organs including the liver, LDL-cholesterols are uptaken through LDL receptormediated endocytosis. LDL receptor (LDLR) is located on the cellular membrane and it recognizes apoB-100 of LDL and carries LDL particles into cells through coated pits. The cholesterol released from LDL can be used or stored, and remaining LDLRs can be either recycled or degraded. It is well known that the synthesis of LDLRs is regulated by SREBP through SRE present on the LDLR promoter (Briggs et al., 1993). The cholesterol levels within the blood throughout the day remain stable regardless of feeding or fasting. However, the well-defined diurnal rhythm of HMG-CoA reductase (Edwards et al., 1972) raises the possibility of circadian expression of LDLR. Indeed, it was once reported that LDLR protein activity displays daily fluctuations in the rat (Balasubramaniam et al., 1994). The purpose of this study is to examine possible circadian regulation of LDLR gene expression and its molecular mecha$\operatorname{nism}(\mathrm{s})$.

\section{Results}

\section{Daily rhythms of metabolic parameters in mice fed ad libitum}

Nocturnal mice consume most of their food during the early scotophase when fed ad libitum (SanchezAlavez et al., 2007). To see whether mice fed ad libitum display daily variation in the metabolic parameters related to cholesterol homeostasis, daily changes in the levels of blood glucose, triglyceride (TG), total cholesterol and HDL cholesterol were determined (Figure 1). Blood glucose levels varied throughout a day, reaching the lowest level in the 

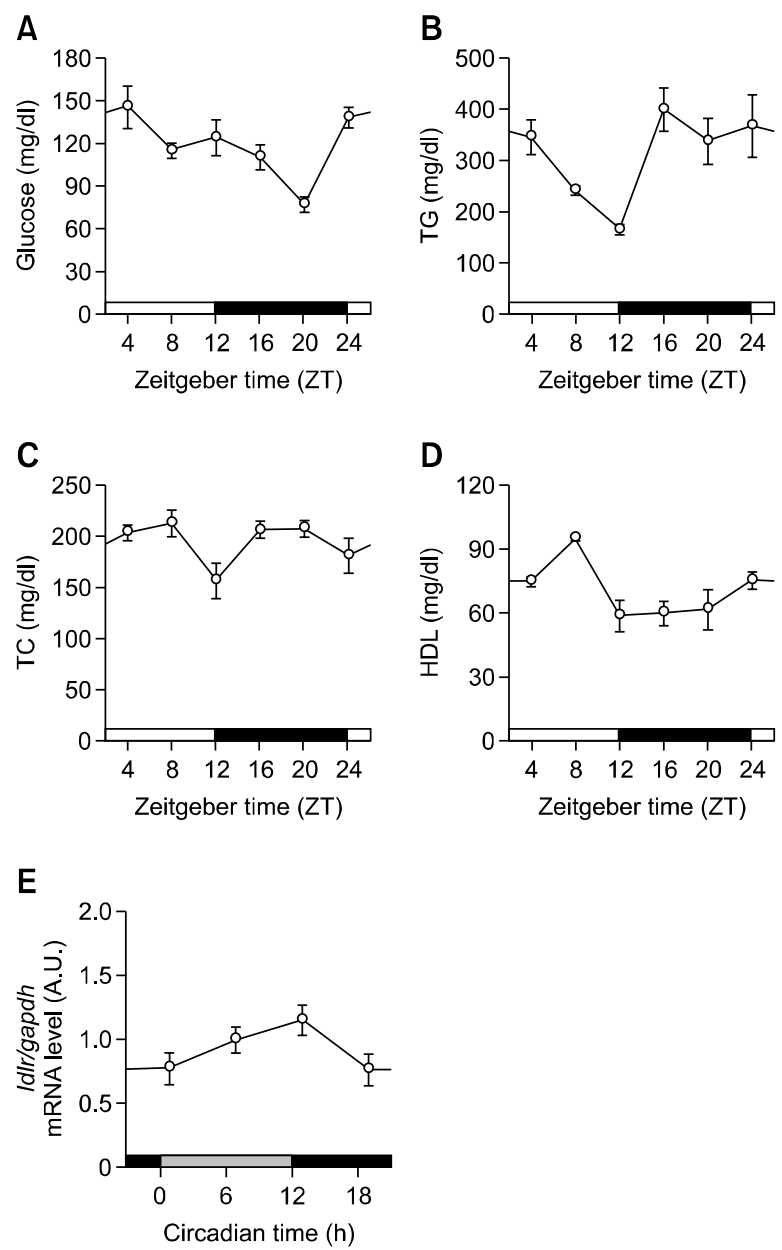

Figure 1. Daily rhythms of metabolic parameters related to cholesterol homeostasis in mice fed ad libitum. (A-D) Male C57BL/6 mice were entrained to 12L:12D cycle for 2 weeks and sacrificed by cervical dislocation at the indicated zeitgeber time (ZT) and whole blood samples were collected. Blood glucose (A), plasma TG (B), total cholesterol (C) and $\mathrm{HDL}$ cholesterol were determined by specific kits obtained from Callegrari $^{\mathrm{TM}}$. Data are expressed as mean \pm S.E.M. $(n=8)$. (E) Oscillation of Idllr mRNA in the mouse liver. Male C57BL/6 mice were entrained to 12L:12D cycle for 2 weeks and released to DD. On the second day after light-off, mice were sacrificed by cervical dislocation at the indicated circadian time and liver samples were obtained. RNA isolation, reverse transcription, and real-time polymerase chain reaction were performed to measure specific messages for mouse Idlr. All mRNA levels were normalized to gapdh mRNA level and expressed as mean \pm S.E.M. $(n=8)$.

middle of active/dark phase (ZT20; Figure 1A). TG and HDL cholesterol levels also displayed distinct daily patterns. TG levels remained high throughout the active phase but declined during the rest reaching lowest level at ZT12 (Figure 1B). Total cholesterol levels remained fairly stable throughout a day but decreased at the time of light-dark transition (Figure 1C). HDL cholesterol remained low during the dark phase but increased during the light phase reaching highest level at ZT8 (Figure
1D). These data indicate that metabolic parameters related to cholesterol homeostasis vary throughout the day in mice fed ad libitum. To see whether Idlr mRNA oscillates in a circadian manner, mouse liver samples were obtained and analyzed every $6 \mathrm{~h}$ (Figure 1E). In the mouse liver, mouse Idlr mRNA displayed circadian rhythm of gene expression having acrophase at around CT12. This is in good agreement with the previous report that LDLR protein reach the highest level at $\mathrm{ZT} 12$ in the rat (Balasubramaniam et al., 1994).

\section{Serum-independent induction by CLOCK/BMAL1 and serum-dependent repression by Hes 1 and Hes 6 of the human IdIr promoter activity}

To investigate the transcriptional regulation of human Idlr, promoter sequences were analyzed. Schematic diagrams of DNA elements on human Idlr gene promoter and serial deletion reporter constructs are presented in Figure 2. The sterol regulatory element (SRE) is located in the proximal region and three putative E-boxes are located within $1 \mathrm{~kb}$ of the upstream promoter. Serial deletion constructs (Jeong et al., 2009) were used to examine responsiveness to CLOCK/BMAL1, Hes1, and Hes6. These constructs were transiently transfected into HepG2 human hepatocarcinoma cells with or without CLOCK/BMAL1, Hes1, or Hes6 expression vectors. Human Idlr gene expression is induced by CLOCK/ BMAL1 in serum-independent manner. However, the inhibitory effects of Hes 1 and Hes 6 were observed only under serum-depleted condition. In the presence of serum, Hes 1 and Hes6 showed no effect. These results indicate that transcriptional regulation of Idlr gene is under circadian control involving CLOCK/BMAL1 and Hes family transcription factors Hes1 and Hes6. To see whether the mouse Idlr gene is also regulated in the same manner, we compared the promoter sequences between mouse and human $I d l r$ genes and generated mouse $I d l r$ promoter constructs as shown in Supplemental Data Figure S1. We found that SRE elements important for the inhibitory effect of Hes 1 and Hes6 are well conserved between human and mouse Idlr genes, while several E-boxes found in distal regions of human and mouse promoters are not so well conserved. Then, we tested whether mouse Idlr gene is also regulated by CLOCK/BMAL1, Hes 1 and Hes6 (Supplemental Data Figure S2). We found that mouse Idlr transcription is also reduced by Hes 1 and Hes6 expression, while the induction by CLOCK/BMAL1 was quite weaker compared with the induction of human constructs (compare Figure S2 with Figures $2 \mathrm{~B}$ and $2 \mathrm{C}$ ), which is in good agreement with the slight increase of mouse LDLR 
A

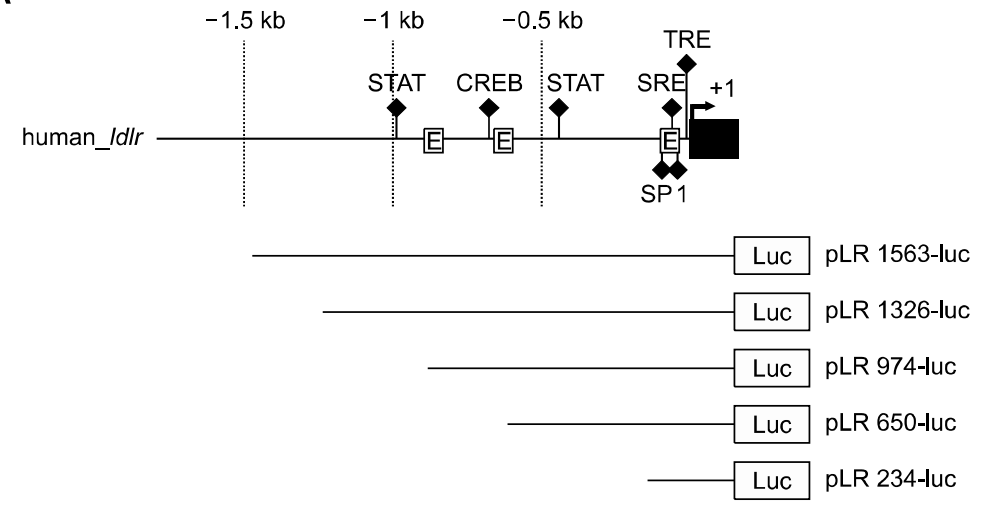

B

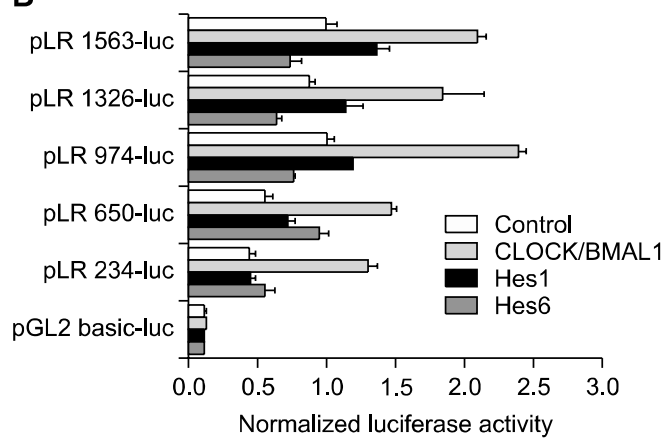

C

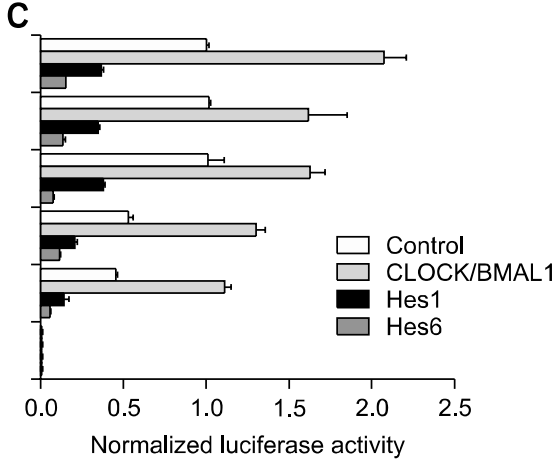

D

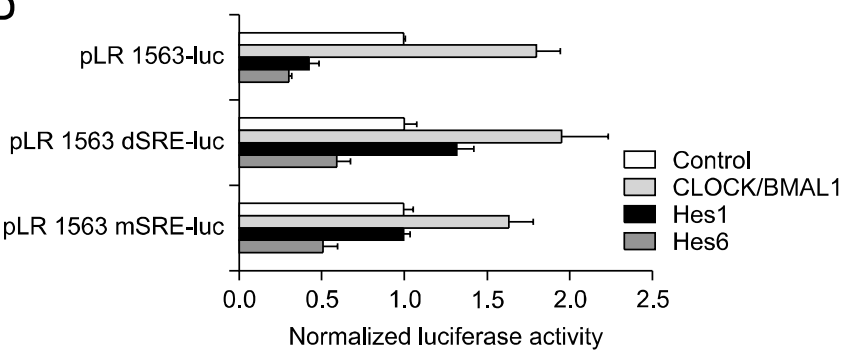

Figure 2. Transcriptional regulation of Idlr promoter activity by CLOCK/BMAL1, Hes1 and Hes6. (A) Schematic diagram of DNA elements on human Idlr gene promoter and serial deletion constructs. Genomic DNA sequences spanning human Idlr (NT_011295, chromosome 19) were analyzed for the transcription factor binding sites using tools provided by Genomatrix (www.genomatrix.de). DNA elements were symbolized as indicated in the figure. The predicted transcription start site is indicated as a bent arrow. Schematic drawing of human Idlr gene reporter constructs is also shown in the lower part of the figure. (B, C) Effect of CLOCK/BMAL1, Hes1 and Hes6 expression on reporter activity. HepG2 human hepatocarcinoma cells were transiently transfected with each reporter construct with or without CLOCK/BMAL1, Hes1, or Hes6 expression vectors. One day after transfection, cells received either 10\% FBS-containing (B) or serum-depleted media (C). Luciferase assay was performed after additional $24 \mathrm{~h}$ incubation. Resulting firefly luciferase activity was normalized to Renilla luciferase activity driven by TK promoter, which was used as an internal control. Data are expressed as mean \pm S.E.M. ( $n=3$ ). (D) The sterol regulatory element (SRE) in the proximal promoter of human Idlr gene is responsible for inhibition by HES1. Effect of CLOCK/BMAL1, Hes1 and Hes6 on reporter activity. HepG2 cells were transiently transfected with pLR 1563-luc or pLR $1563 \mathrm{mSRE}-l \mathrm{luc}$ or pLR $1563 \mathrm{dSRE-luc} \mathrm{construct} \mathrm{with} \mathrm{or}$ without CLOCK/BMAL1, Hes1 and Hes6 expression vectors. Resulting firefly luciferase activity was normalized to Renilla luciferase activity driven by TK promoter, which were used as an internal control. Data are expressed as mean \pm S.E.M.

mRNA around CT12 (Figure 1E). Moreover, the induction by CLOCK/BMAL1 was blunted with the proximal promoter construct (-263 mldlr-Luc), while the repression by Hes 1 and Hes6 still remained. Surprisingly, in contrast to human promoters (Figures $2 \mathrm{~B}$ and $2 \mathrm{C}$ ), reduction of mouse $/ d$ lr r transcription by
Hes 1 and Hes6 were observed also in serumenriched condition. Thus, there exist some convergent and divergent controls of Idlr gene transcriptions between human and mouse promoters. 
A Wild type
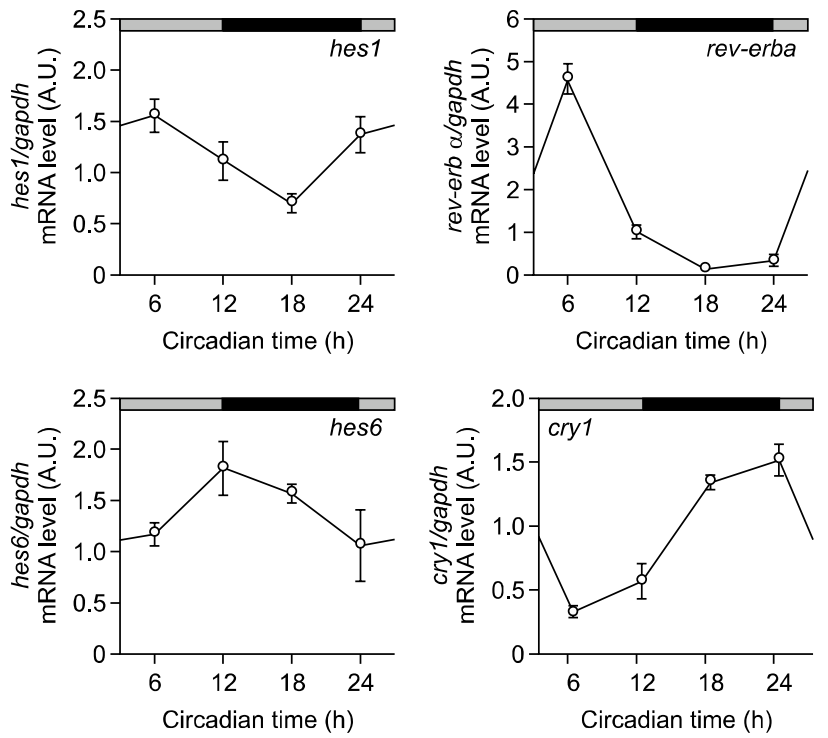

B Per1 ${ }^{-/-}$Per2 $2^{-/-}$
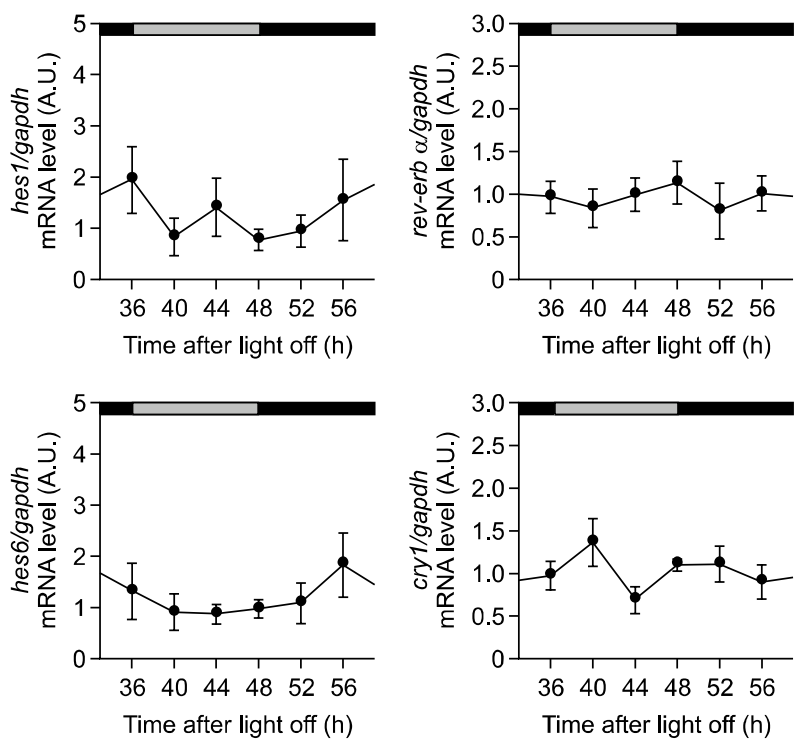

\section{The sterol regulatory element (SRE) mediates the Hes1-induced transcriptional repression}

The serum-dependent inhibitory effect of Hes1 and Hes6 suggests involvement of SRE. To test this possibility, SRE-mutated (pLR 1563 mSRE-luc) or SRE-deleted (pLR 1563 dSRE-luc) Idlr reporter constructs were used. These constructs were transiently transfected into HepG2 cells with or without CLOCK/BMAL1, Hes1, or Hes6 expression vectors under serum-depleted condition (Figure 2D). Interestingly, inhibitory effect of Hes1 was abolished but still induced by CLOCK/BMAL1 and repressed by Hes6. These results indicate that Hes1-induced
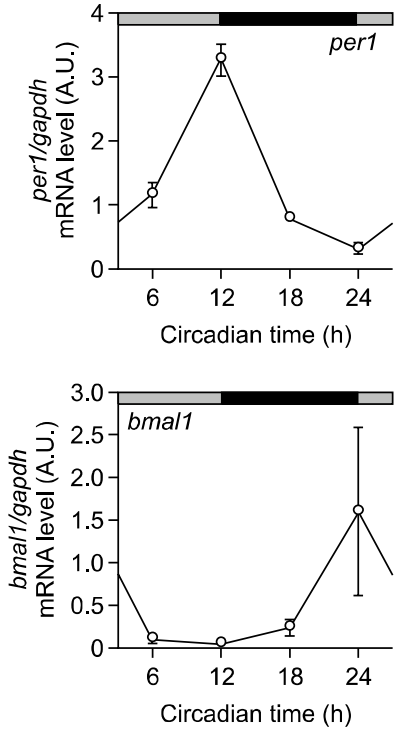

Figure 3. Anti-phasic oscillation of hes1 and hes6 mRNA in the wild-type (A) but not in per1 ${ }^{-1 /}$ per2 (B) mouse liver. Male C57BL/6 mice of each genotype were entrained to 12L:12D cycle for 2 weeks and released to DD. On the second day after light-off, mice were sacrificed by cervical dislocation at the indicated circadian time and liver samples were obtained. RNA isolation, reverse transcription, and real-time polymerase chain reaction were performed to measure specific messages for rev-erba, cry1, bmal1b, hes6 and hes1. All mRNA levels were normalized to gapdh mRNA level and expressed as mean \pm S.E.M. $(n=3)$

transcriptional repression is mediated by SRE and the Hes6-induced transcriptional repression may involve other element(s).

\section{Anti-phasic oscillation of hes6 and hes 1 genes expression in the mouse liver}

To see whether hes 6 and hes 1 gene expressions are also controlled by circadian clock, hes 6 , hes 1 and various clock genes expressions were analyzed in both WT and circadian clock mutant mice. Wild type and per1 $1^{-/}$per2 ${ }^{-/-}$mice (Park et al., 2012) were first entrained to a 12L:12D lighting cycle for 2 
A

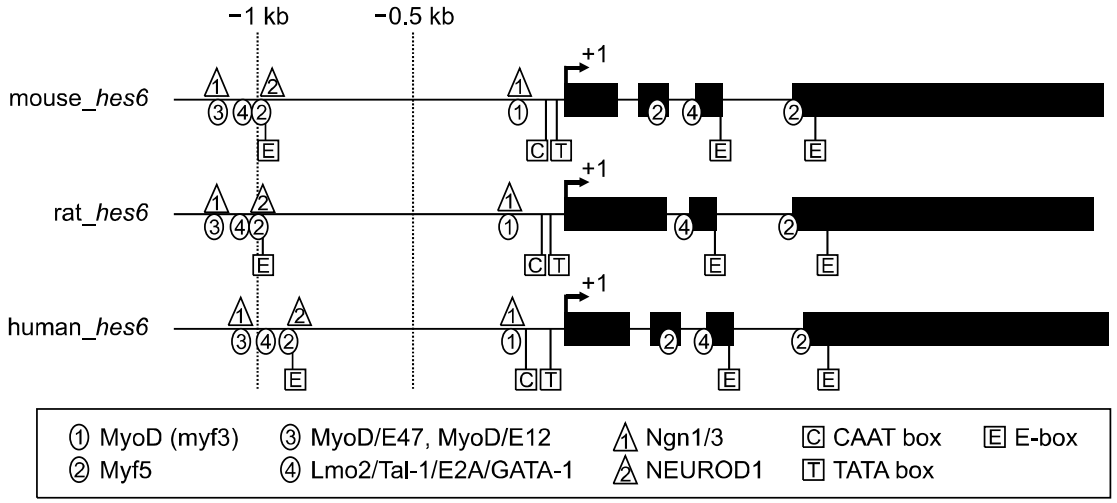

B

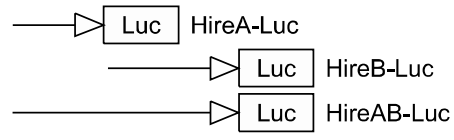

$\mathrm{m}$ hes6
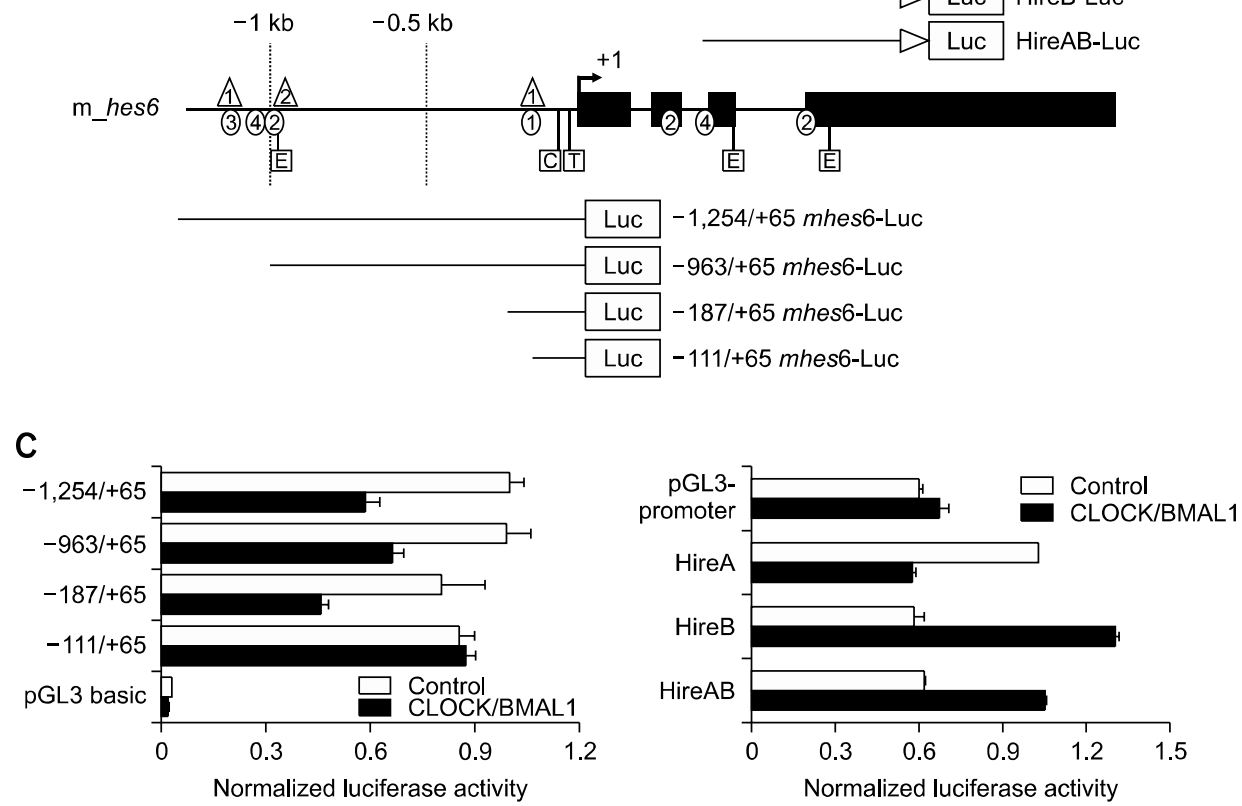

D
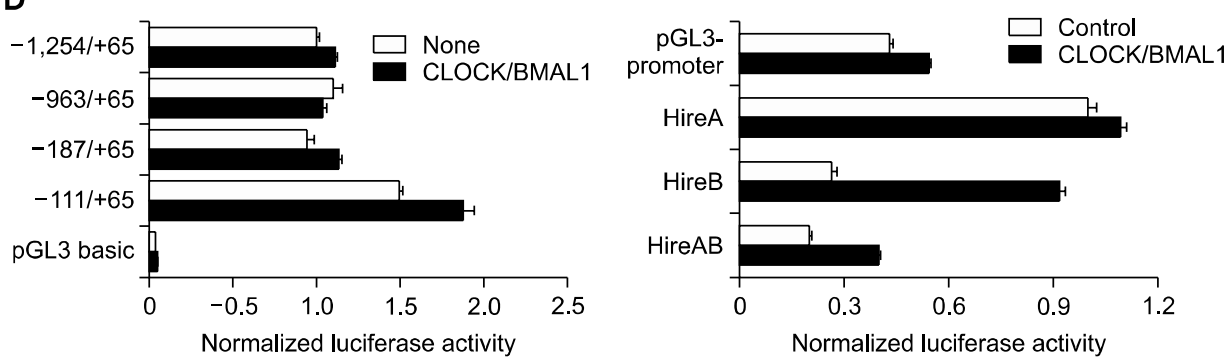

Figure 4. Mouse hes6 gene is induced by CLOCK/BMAL1 via downstream regulatory sequence. (A) Schematic diagrams of mouse, rat, and human hes6 gene structure and conserved DNA elements. Genomic DNA sequences spanning mouse (Accession No. NT078297, chromosome 1), rat (NW 047817 , chromosome 9), and human hes6 (NT_005120, chromosome 2) were analyzed for the common transcription factor binding sites using tools provided by Genomatrix (www.genomatrix.de). Evolutionarily conserved DNA elements were symbolized as indicated in the figure. (B) Schematic drawing of mouse hes6 gene reporter constructs. The four serial deletion constructs were made from upstream promoter region of hes 6 and regulatory elements were made from intergenic region. See Materials and Methods for details. The right headed triangle indicates SV40 promoter. (C, D) Effect of CLOCK/BMAL1 expression on reporter activity. NIH 3T3 mouse fibroblast (C) and HT22 mouse hippocampal cells (D) were transiently transfected with each reporter construct with or without CLOCK/BMAL1 expression vectors. Resulting firefly luciferase activity was normalized to Renilla luciferase activity driven by TK promoter, which was used as an internal control. Data are expressed as mean \pm S.E.M. 
weeks and released to constant darkness (DD). On the second day after light-off, mouse liver samples were obtained every $6 \mathrm{~h}$ from CT6. The mRNA levels of the liver samples were analyzed using real-time RT-PCR (Figure 3). The core clock, rev-erba, per1, cry1, and bmal1b, displayed clear circadian rhythm of gene expression having acrophases at around CT6, CT12, CT18-24, and CT24, respectively. The hes 6 mRNA expression also showed circadian rhythm having acrophase at around CT18 in the middle of per1 and cry1, while hes1 mRNA expression having the lowest value at CT18. Moreover, all of these rhythms disappeared in per $1^{-1-}$ per $2^{-1-}$ mouse liver. These results suggest that hes 6 and hes 1 are clock controlled genes (ccgs) that may have roles in the mouse liver.

\section{The hes 6 gene structure and conserved DNA elements}

To investigate the molecular mechanism allowing circadian expression of hes6, mouse, rat and human hes 6 were analyzed and summarized in Figure 4A. The mouse and human hes 6 genes are composed of four exons and three introns, while rat hes 6 is composed of three exons and two introns. The hes 6 promoters up to $1.2 \mathrm{~kb}$ are well conserved among three species: Especially, Ngn1/3, NeuroD1, MyoD binding sites important for neurogenesis or myogenesis are placed forming clusters in two distinct locations of promoter region (about $-1 \mathrm{~kb}$ or $-0.2 \mathrm{~kb}$ region). This conforms to the findings that hes 6 is mainly regulated during neurogenesis and myogenesis (Bae et al., 2000; Murai et al., 2007). Interestingly, three E-boxes, putative binding sites for positive circadian transcription factor complex CLOCK/BMAL1 were found conserved: one on $\sim-0.9 \mathrm{~kb}$, and the other two on inton 3 and exon IV. This raises the possibility that hes6 may be regulated by CLOCK/BMAL1.

\section{Regulation of hes 6 gene transcription by CLOCK/BMAL1}

Several constructs were generated to examine the possible regulation of hes 6 gene transcription by CLOCK/BMAL1 (Figure 4B). The serial deletional constructs were made from mouse hes 6 promoter region and regulatory constructs were from intragenic sequence. In the upstream reporters, the longest construct, -1254/+65-Luc, has an E-box and in the regulatory constructs HireA-LuC and HireB-Luc have one E-box each and Hire AB-Luc has two downstream E-boxes. These constructs were transiently co-transfected with CLOCK/BMAL1 expression vectors into NIH 3 T3 cells and HT22
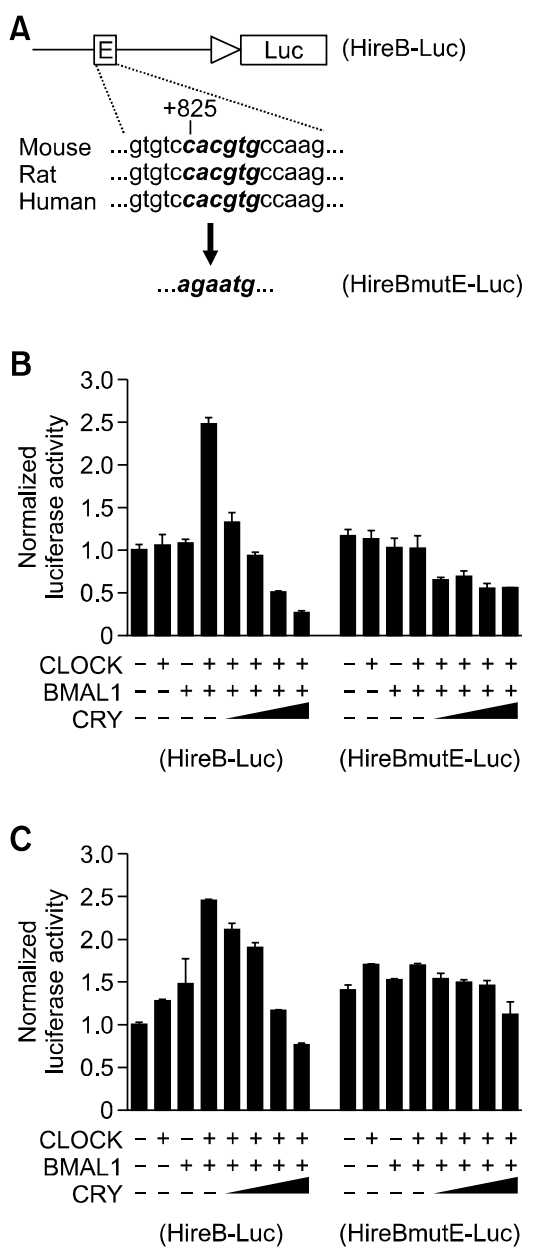

Figure 5. Conserved E-box in the exon IV of mouse hes6 gene is responsible for induction by CLOCK/BMAL1 and inhibition by CRY1. (A) Location of conserved E-box in HireB-Luc construct and point mutation resulting in HireBmut-Luc construct. Large triangle indicates SV40 promoter. (B, C) Effect of CLOCK/BMAL1 and increasing amount of CRY1 expression on reporter activity. NIH $3 \mathrm{~T} 3$ mouse fibroblasts (B) and HT22 mouse hippocampal cells $(C)$ were transiently transfected with either HireB-Luc or HireBmutE-Luc construct with or without CLOCK/BMAL1 and CRY1 expression vectors. Resulting firefly luciferase activity were normalized to Renilla luciferase activity driven by TK promoter, which were used as an internal control. Data are expressed as mean \pm S.E.M.

cells (Figures $4 \mathrm{C}$ and $4 \mathrm{D}$ ). The significant and reproducible induction by CLOCK/BMAL1 appeared only in HireB-Luc and HireAB-Luc constructs. This result indicates that the regulatory sequence containing exonic E-box is responsible for the induction by CLOCK/BMAL1.

\section{The functionality of exonic E-box}

To confirm the functionality of E-box on exon IV, point mutation studies were performed. The E-box sequence of HireB-Luc, CACGTG, was mutated to 
AGAATG (Figure 5) using HireB-Luc as a template. The WT and mutated constructs were transiently co-transfected with CLOCK/BMAL1 and with or without CRY expression vector into NIH 3T3 and HT22 cells (Figures 5B and $5 \mathrm{C}$ ). The induction by CLOCK/BMAL1 and repression by CRY1 observed in the HireB-Luc disappeared in the HireBmutE-Luc construct. Chromatin immunoprecipitation also demonstrated the binding of BMAL 1 to this region in vivo (data not shown). These results clearly indicate that E-box on exon IV is functional.

\section{Discussion}

The molecular clockwork plays important roles in diverse physiological and pathological settings by controlling timed expression of other regulatory and/or structural genes (reviewed in Takahashi et al., 2008). Especially notable is metabolic clockwork which has great implications in diverse human pathologies including metabolic syndrome (reviewed in Green et al., 2008). The present work demonstrating the role of CLOCK/BMAL1, Hes1 and Hes6 in the LDLR expression suggests multiple and complex regulation of LDLR at the transcription level.

The daily changes of LDLR protein level in the rat was reported previously (Balasubramaniam et al., 1994) and this work provides the possible mechanism underlying it. Using HepG2 human hepatocarcinoma cells, we demonstrated that CLOCK/BMAL1 induces $l d l r$ transcription in a serum-independent manner (Figure 2; see also Figure S2). Accordingly, Idlr mRNA in the wild-type mouse liver peaked around CT12 (Figure 2) at the time other E-box regulated genes such as per1 and cry1 are also upregulated (Figure 3). This implies that LDLR is one of the clock-controlled genes and elevates LDLR expression before major mealtime in mice (Sanchez-Alavez et al., 2007; Yoon et al., 2012).

In the present study, Hes family members Hes1 and Hes6 downregulated human LDLR expression only under serum-depleted conditions in HepG2 cells (Figures 2B and 2C), although they downregulated mouse LDLR in a serum-independent fashion (Figure S2). This indicates that the upregulation of LDLR in the absence of serum or cholesterol (Jeong et al., 2009 and references therein) can be efficiently blocked by Hes 1 and Hes6 whose expressions are also under the control of circadian clock and involve distinct promoter elements of LDLR. The convergent and divergent promoter structures and/or differential control of human and mouse Idlr genes (Figures S1 and S2) are quite intriguing and necessitate more extensive works.

LDLR is a cell-surface glycoprotein that serves in the homeostatic control of blood cholesterol by mediating the elimination of cholesterol-containing lipoprotein particles from blood circulation (Brown and Goldstein, 1986). In case of sterol depletion, the Idlr transcription markedly increases, while it decreases in case of sterol accumulation (Goldstein and Brown, 1990; Ellsworth et al., 1994). It is known that serum depletion or lipoprotein deficiency powerfully induces the LDLR expression in cultured HepG2 cells (Pak et al., 1996). Sterol-mediated repression or induction of $l d l r$ promoter is mediated by sterol regulatory element-1 (SRE-1) (Smith et al., 1990), which has been shown to bind SRE-BP-1 (SRE-binding protein-1) and SRE-BP-2 (SRE-binding protein-2) (Briggs et al., 1993; Wang et al., 1993). In the absence of sterol, proteases cleave the SRE-BP, which allows translocation of the truncated SREBP to the nucleus where it activates transcription of the LDL receptor.

The present study showed that Hes1 exerts its effect through sterol regulatory element of the LDLR promoter (Figure 2) which is bound by SRE-BP when intracellular cholesterol level goes lower. Thus, the possible interference of Hes1 with SRE-BP action is an intriguing possibility that needs to be resolved in the future. In contrast, the action of Hes6 is independent of SRE but involves the similar proximal region of the LDLR promoter. So far, it was fruitless to delineate the regulatory element(s) that is responsible for it. As Hes6 is known to interfere with other transcription factors such as Hes 1 and MyoR under different cellular context, an indirect action of Hes6 remains an open question.

The present work analyzed mouse, rat, and human hes 6 genes promoter and coding sequences and found that many transcription factor binding sites important for neurogenesis and myogenesis are well conserved as expected (Figure 4). Moreover, a fully functional E-box is found within the exon IV of the hes 6 gene as demonstrated by point mutation (Figure 5) and chromatin immunoprecipitation assay (data not shown). Accordingly, hes 6 gene expression oscillated in an anti-phasic manner with hes 1 in the mouse liver in a functional clockdependent manner (Figure 3) and seems to be involved in the regulation of LDLR. Although aberrant expression of Notch signaling genes including Hes1 and Hes6 was reported in the hepatocellular carcinogenesis (Gramantieri et al., 2007), this is the first demonstration that Hes6 and Hes 1 may have a functional role in the liver regulating cholesterol homeostasis.

The present work poses critical implications for 
human metabolic diseases (Al Rayyes et al., 1997; Hazra et al., 2008). For instance, the human genetic disease called familial hypercholesterolemia $(\mathrm{FH})$ is caused by genetic defects in the LDLR. The FH patients possess increased risk of high blood cholesterol and display myocardial infarctions (heart attacks), orange-yellow xanthomas, atherosclerosis, and cardiovascular diseases (Brown and Goldstein, 1986; Goldstein and Brown, 2009). Thus, the aberrant expression and dysregulation of LDLR in the absence of functional clock, hes1, or hes 6 in vivo is an intriguing possibility that is under investigation.

In modern society, more and more workers are involved in various kinds of shift works. Epidemiological evidence indicates that shift works are closely associated with increased risk for metabolic syndrome (Szosland, 2010). Moreover, an animal model of circadian disturbance increased mortality in mice (Park et al., 2012). Low density lipoprotein receptor (LDLR) is known to play a key role in cholesterol metabolism that has been associated with an increased risk of coronary heart disease and atherosclerosis (Brown and Goldstein, 1986). Thus, together with our recent observation that meal time shift disturbs circadian rhythmicity and metabolic homeostasis in mice (Yoon et al., 2012), possible circadian regulation of LDLR promoter by CLOCK/BMAL1, Hes1 and Hes6 poses great implications for human health and mortality.

\section{Methods}

\section{Chemicals and reagents}

Unless indicated otherwise, all the chemicals and reagents were obtained from Sigma.

\section{Animals, entrainment, and circadian sampling}

All animal experiments were approved and performed under the guidelines of Kyung Hee University Institutional Animal Care and Use Committee. PDK mice have been described elsewhere (Park et al., 2012). All cages were placed and maintained in a light-proof Clean Animal Rack cabinet (Shin Biotech, Seoul, Korea) with constant air ventilation. Lighting schedule was automatically controlled by equipped digital timers and the ambient temperature was maintained at $23 \pm 1^{\circ} \mathrm{C}$. Light intensity during the light phase was maintained at 350-450 lux throughout the area. Adult wild-type and per1 $1^{-1-}$ per2 ${ }^{-/-}$male mice were entrained to a 12:12 LD photoperiodic cycle with light-on at 08:00 AM. After 2 weeks of entrainment, animals were released to constant darkness (DD). On the second day after light-off, animals were sacrificed and liver samples were obtained at the indicated circadian time.

\section{Blood collection and measurements of glucose and cholesterol in the blood}

8 week-old wild-type male mice were entrained to a 12:12 LD photoperiodic cycle for 2 weeks and sacrificed by cervical dislocation at the indicated zeitgeber time (ZT) and whole blood samples were collected into tubes containing heparin. Blood glucose levels were determined using Accu-Chek ${ }^{\circledR}$ Performa kit (Roche) at the time of sacrifice. Remaining blood samples were centrifuged at 3,000 rpm at $4^{\circ} \mathrm{C}$ for $10 \mathrm{~min}$. Triglyceride, total cholesterol and HDL cholesterol levels in the upper plasma were determined using specific kits and CR-3000 apparatus from Callegrari ${ }^{\mathrm{TM}}$ according to the manufacturer's specifications (Yoon et al., 2012).

\section{Promoter analysis}

Genomic DNA sequences spanning exons, introns, and 3 $\mathrm{Kb}$ upstream promoter region of mouse (Accession No. NT078297, chromosome 1), rat (NW_047817, chromosome 9), and human hes6 (NT_005120, chromosome 2) were accessed at NCBI website (http://www.ncbi.nlm.nih.gov). These sequences were analyzed for the common transcription factor binding sites using tools provided by Genomatrix (www.genomatrix.de). Conserved DNA elements were found and used as a reference for the generation of reporter constructs.

\section{Cloning of reporter constructs}

The LDLR reporter constructs were described elsewhere (Jeong et al., 2009). The serial deletion reporter constructs $(-1254,-963,-187,-111 /+65$ mhes6-Luc) and intronic regulatory element reporter constructs (HireA, HireB, HireAB-Luc) of hes6 were generated by PCR with each set of primers with Kpnl or Nhel linker as diagramed in Figure 4. PCR products were cloned into the pGL3-basic or pGL3-promoter vector (Promega) at $\mathrm{Kpnl} / \mathrm{Nhel}$ site. Correct cloning was verified by restriction enzyme cutting and subsequent DNA sequencing. Primer sequences used are as follows: H6up1(Kpnl linker): atg gta cca aat cca aga acg ctg gag c; H6up2 (Kpnl linker): atg gta cca agt tac tgg ggc cag aca g; H6up3 (Kpnl linker): atg gta cct aag tgg cag gag gtc tgg c; H6up4 (Kpnl linker): atg gta ccc tct cat tgg cta cag gct g; H6dn5 (Nhel linker): atg cta gcc tag tgc aaa cta ctc agc g; HireAup (Kpnl linker): atg gta cct ctg cgc agg tgc agg cca a; HireBdn (Nhel linker): atg cta gca agc cct tgc cat gga tag g; HireCup (Kpnl linker): atg gta cct tcc cac cct cct gca cag; HireDdn (Nhel linker): atg cta gct ctg gga tct cct cta ggt c.

\section{Cell culture, transient transfection, and dual luciferase reporter assay}

HT22 mouse hippocampal cells, NIH 3T3 mouse embryonic fibroblast cells, and HepG2 human hepatocarcinoma cells were cultivated in DMEM supplemented with $10 \%$ FBS and $100 \mathrm{U} / \mathrm{ml}$ penicillin and $100 \mu \mathrm{g} / \mathrm{ml}$ streptomycin in a humidified atmosphere containing $5 \% \mathrm{CO}_{2}$ at $37^{\circ} \mathrm{C}$ (Doi et al., 2007; Park and Pak, 2011). For transient transfection, cells were seeded in 24-well plates at a density of $5 \times 10^{4}$ per well. On the following day, cells were trans- 
fected with each of the luciferase reporter construct and expression vectors using lipofectamine reagent (Invitrogen). To compensate transfection efficiency, $20 \mathrm{ng}$ of the minimal thymidine kinase promoter-driven Renilla luciferase construct ( $p R L-m T K$ ) was cotransfected. The total amount of plasmid was held constant by adding pcDNA3 plasmid as a carrier DNA. The plasmids used for transfection were prepared using QIAGEN miniprep kit. After $48 \mathrm{~h}$ of transfection, cells were lysed and luciferase activity was measured using dual lucifease reporter assay kit (Promga) and DLReady Berthold luminometer (Centro LB960) according to the manufacturer's specifications. The firefly luciferase activity was normalized to Renilla luciferase activity and presented as relative values of the control transfection.

\section{Point mutation of reporter constructs}

in vitro mutagenesis of conserved E-box to generate HireBmutE-Luc (CACGTG $\rightarrow$ AGAATG) was performed on the HireB-Luc construct using Quick Change Site-Directed Mutagenesis kit (Stratagene). The primer set for mutated E-box is 5'-CAC ACG TTC GTG TCA GAA TGC CAA GCC ATC GAT $G$ and 5'-CAT CGA TGG CTT GGC ATT CTG ACA CGA ACG TGT G.

\section{RNA isolation, reverse transcription and real-time PCR}

Total RNA was isolated from tissues and cells using acid guanidinium phenol-chloroform (AGPC) method (Cho et al., 1998). One $\mu \mathrm{g}$ of total RNA was reverse transcribed using RTase M-MLV (Takara, 2640A) with $200 \mathrm{ng}$ of random hexamer, $2.5 \mathrm{mM}$ each of dNTP and RNase inhibitor (Takara, 2310A) at $37^{\circ} \mathrm{C}$ for $1 \mathrm{~h}$. The procedure for real-time PCR using LightCycler has been described elsewhere (Doi et al., 2007). Briefly, the standard was prepared by pooling 5 -fold diluted RT samples in $1 \mathrm{mM}$ Tris. The templates for real-time PCR were prepared by further 15 -fold dilution of 5-fold diluted RT samples. 2X SYBR Premix EX Taq (Takara, RR041A) and LightCycler Version 1.5 (Roche, Salt Lake City) were used for the real-time PCR. The expression levels of gapdh were used for normalization. Primer sequences used for real-time PCR are summarized in Supplemental Data Table S1.

\section{Supplemental data}

Supplemental data include two figures and a table and can be found with this article online at http://e-emm.or.kr/article/ article_files/SP-44-11-02.pdf.

\section{Acknowledgements}

We thank Dr. Jaesang Kim (Ewha Woman's University, Seoul, Korea) and Dr. Woong Sun (Korea University, Seoul Korea) for kind donations of Hes1 and Hes6 expression vectors, respectively. This work was supported by a grant funded by Kyung Hee University (No. 20060491).

\section{References}

Al Rayyes $\mathrm{O}$, Wallmark $\mathrm{A}$, Floren $\mathrm{CH}$. Additive inhibitory effect of hydrocortisone and cyclosporine on low-density lipoprotein receptor activity in cultured HepG2 cells. Hepatology 1997;26:967-71

Bae S, Bessho Y, Hojo M, Kageyama R. The bHLH gene Hes6, an inhibitor of Hes1, promotes neuronal differentiation. Development 2000;127:2933-43

Balasubramaniam S, Szanto A, Roach PD. Circadian rhythm in hepatic low-density-lipoprotein (LDL)-receptor expression and plasma LDL levels. Biochem J 1994;298:39-43

Briggs MR, Yokoyama C, Wang X, Brown MS, Goldstein JL. Nuclear protein that binds sterol regulatory element of low density lipoprotein receptor promoter. I. Identification of the protein and delineation of its target nucleotide sequence. $J$ Biol Chem 1993;268:14490-6

Brown MS, Goldstein JL. A receptor-mediated pathway for cholesterol homeostasis. Science 1986;232:34-47

Cho S, Cho H, Geum D, Kim K. Retinoic acid regulates gonadotropin-releasing hormone $(\mathrm{GnRH})$ release and gene expression in the rat hypothalamic fragments and GT1-1 neuronal cells in vitro. Brain Res Mol Brain Res 1998;54: 74-84

Doi M, Cho S, Yujnovsky I, Hirayama J, Cermakian N, Cato AC, Sassone-Corsi P. Light-inducible and clock-controlled expression of MAP kinase phosphatase 1 in mouse central pacemaker neurons. J Biol Rhythms 2007;22:127-39

Dunlap JC. Molecular bases for circadian clocks. Cell 1999; 96:271-90

Edwards PA, Muroya H, Gould RG. In vivo demonstration of the circadian rhythm of cholesterol biosynthesis in the liver and intestine of the rat. J Lipid Res 1972;13:396-401

Ellsworth JL, Carlstrom AJ, Deikman J. Ketoconazole and 25-hydroxycholesterol produce reciprocal changes in the rate of transcription of the human LDL receptor gene. Biochim Biophys Acta 1994;1210:321-8

Goldstein JL, Brown MS. Regulation of the mevalonate pathway. Nature 1990;343:425-30

Goldstein JL, Brown MS. The LDL receptor. Arterioscler Thromb Vasc Biol 2009; 29:431-8

Gramantieri L, Giovannini C, Lanzi A, Chieco P, Ravaioli M, Venturi A, Grazi GL, Bolondi L. Aberrant Notch3 and Notch4 expression in human hepatocellular carcinoma. Liver Int 2007;27:997-1007

Green CB, Takahashi JS, Bass J. The meter of metabolism. Cell 2008;134:728-42

Ha M, Park J. Shiftwork and metabolic risk factors of cardiovascular disease. J Occup Health 2005;47:89-95

Hazra A, Pyszczynski NA, DuBois DC, Almon RR, Jusko WJ. Modeling of corticosteroid effects on hepatic low-density lipoprotein receptors and plasma lipid dynamics in rats. Pharm Res 2008;25:769-80

Ishida $\mathrm{A}$, Mutoh $\mathrm{T}$, Ueyama $\mathrm{T}$, Bando $\mathrm{H}$, Masubuchi $\mathrm{S}$, Nakahara D, Tsujimoto G, Okamura H. Light activates the 
adrenal gland: timing of gene expression and glucocorticoid release. Cell Metab 2005;2:297-307

Jeong JH, Cho S, Pak YK. Sterol-independent repression of low density lipoprotein receptor promoter by peroxisome proliferator activated receptor $\gamma$ coactivator-1a (PGC-1 $\alpha$ ). Exp Mol Med 2009;41:406-16

$\mathrm{Ko} \mathrm{CH}$, Takahashi JS. Molecular components of the mammalian. Hum Mol Genet 2006;15:R271-7

Knutsson A. Health disorders of shift workers. Occup Med (Lond) 2003;53:103-8

Moore RY. Organization and function of a central nervous system circadian oscillator: The suprachiasmatic hypothalamic nucleus. Fed Proc 1983;42:2783-9

Murai K, Vernon AE, Philpott A, Jones P. Hes6 is required for MyoD induction during gastrulation. Dev Biol 2007;312: 61-76

Noh JY, Han DH, Yoon JA, Kim MH, Kim SE, Ko IG, Kim KH, Kim CJ, Cho S. Circadian rhythms in urinary functions: possible roles of circadian clocks? Int Neurourol J 2011;15: 64-73

Panda S, Hogenesch JB. It's all in the timing: many clocks, many outputs. J Biol Rhythms 2004;19:374-87

Pak YK, Kanuck MP, Berrios D, Briggs MR, Cooper AD, Ellsworth JL. Activation of LDL receptor gene expression in HepG2 cells by hepatocyte growth factor. J Lipid Res 1996; 37:985-98

Park WH, Pak YK. Insulin-dependent suppression of cholesterol $7 \alpha$-hydroxylase is a possible link between glucose and cholesterol metabolisms. Exp Mol Med 2011;43: 571-9

Park N, Cheon S, Son GH, Cho S, Kim K. Chronic circadian disturbance by a shortened light-dark cycle increases mortality. Neurobiol Aging 2012;33:1122.e11-22

Roenneberg T, Merrow M. The network of time: understanding the molecular circadian system. Curr Biol 2003;13: R198-207
Sanchez-Alavez M, Klein I, Brownell SE, Tabarean IV, Davis CN, Conti B, Bartfai T. Night eating and obesity in the EP3R-deficient mouse. Proc Natl Acad Sci USA 2007;104: 3009-14

Smith JR, Osborne TF, Goldstein JL, Brown MS. Identification of nucleotides responsible for enhancer activity of sterol regulatory element in low density lipoprotein receptor gene. J Biol Chem 1990;265:2306-10

Stokkan KA, Yamazaki S, Tei H, Sakaki Y, MenakerM. Entrainment of the circadian clock in the liver by feeding. Science 2001;291:490-3

Szosland D. Shift work and metabolic syndrome, diabetes mellitus and ischaemic heart disease. Int $\mathrm{J}$ Occup Med Environ Health 2010;23:287-91

Takahashi JS, Hong HK, Ko CH, McDearmon EL. The genetics of mammalian circadian order and disorder: implications for physiology and disease. Nat Rev Genet 2008:9:764-75

Turek FW, Joshu C, Kohsaka A, Lin E, Ivanova G, McDearmon E, Laposky A, Losee-Olson S, Easton A, Jensen DR, Eckel RH, Takahashi JS, Bass J. Obesity and metabolic syndrome in circadian clock mutant mice. Science 2005;308:1043-5

Wang X, Briggs MR, Hua X, Yokoyama C, Goldstein JL, Brown MS. Nuclear protein that binds sterol regulatory element of low density lipoprotein receptor promoter. II. Purification and characterization. J Biol Chem 1993;268: 14497-504

Whitmer RA, Gustafson DR, Barrett-Connor E, Haan MN, Gunderson EP, Yaffe K. Central obesity and increased risk of dementia more than three decades later. Neurology 2008; 71:1057-64

Yoon JA, Han DH, Noh JY, Kim MH, Son GH, Kim K, Kim K, Pak YK, Cho S. Meal time shift disturbs circadian rhythmicity with metabolic and behavioral alterations in mice. PLoS One 2012;7:e44053 